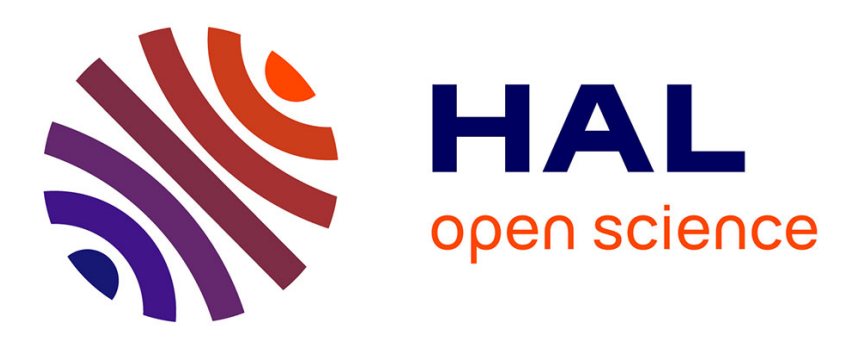

\title{
Towards Road Traffic Management with Forecasting on Wall Displays
}

\author{
Arnaud Prouzeau, Anastasia Bezerianos, Olivier Chapuis
}

\section{To cite this version:}

Arnaud Prouzeau, Anastasia Bezerianos, Olivier Chapuis. Towards Road Traffic Management with Forecasting on Wall Displays. Proceedings of the 2016 International Conference on Interactive Surfaces and Spaces, Nov 2016, Niagara Falls, Canada. pp.119-128, 10.1145/2992154.2992158 . hal01370231v2

\section{HAL Id: hal-01370231 \\ https://hal.science/hal-01370231v2}

Submitted on 15 Nov 2016

HAL is a multi-disciplinary open access archive for the deposit and dissemination of scientific research documents, whether they are published or not. The documents may come from teaching and research institutions in France or abroad, or from public or private research centers.
L'archive ouverte pluridisciplinaire HAL, est destinée au dépôt et à la diffusion de documents scientifiques de niveau recherche, publiés ou non, émanant des établissements d'enseignement et de recherche français ou étrangers, des laboratoires publics ou privés. 


\title{
Towards Road Traffic Management with Forecasting on Wall Displays
}

Arnaud Prouzeau

\author{
Anastasia Bezerianos
}

LRI, Univ Paris-Sud, CNRS, Inria, Universi

F-91405 Orsay, France

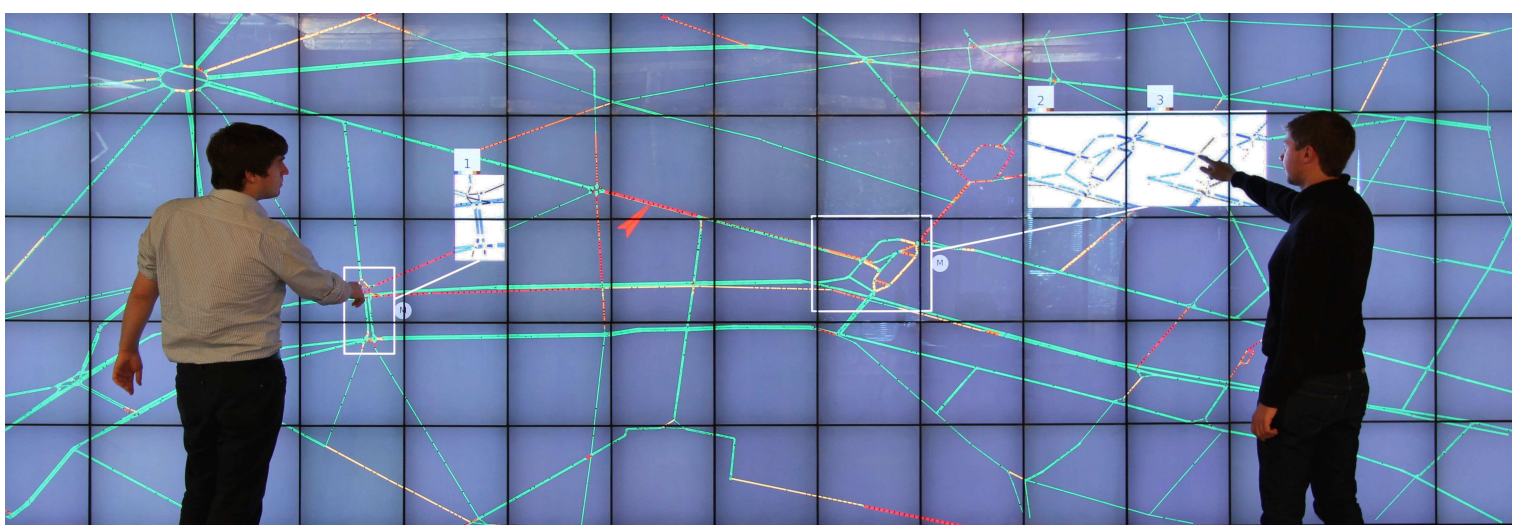

Figure 1. Visualization of traffic in a city with two "DragMagics" (white rectangles) showing one (left) and two (right) simulations associated with different possible interventions on the traffic. The simulation visualizations use difference color maps to highlight differences with the real traffic.

\section{ABSTRACT}

Road traffic control centers are of vital importance to modern cities. Interviews with controllers in two such centers identified the need to incorporate the visualization of results from predictive traffic models with real traffic, to help operators choose among different interventions on the network. We explore this idea in a prototype that runs on a wall display, and supports direct touch and input from workstations and mobile devices. Apart from basic functionality to manage the current traffic such as changing traffic light duration or speed limits, the prototype incorporates traffic simulations for forecasting results of possible actions, highlighting their differences to current traffic. Based on needs identified in our interviews, we offer two techniques that visually combine simulated and real situations, taking advantage of the large display space: multiple independent views and DragMagic, a variation of magic lenses. A preliminary laboratory experiment suggests that both techniques are viable design options, even for monitoring several simulations and areas of interest, contrary to expectations from previous work. However DragMagics are easier to master. An informal feedback session with our experts showed promising early feedback.

\section{Author Keywords}

Traffic monitoring; wall display; road traffic model; traffic forecast; DragMag; magic lens

\footnotetext{
Arnaud Prouzeau, Anastasia Bezerianos \& Olivier Chapuis. Towards Road Traffic Management with Forecasting on Wall Displays, In ISS '16: Proceedings of the 2016 International Conference on Interactive Surfaces and Spaces, 119-128, ACM, November 2016.

(C) ACM, 2016. This is the author's version of the work. It is posted here by permission of ACM for your personal use. Not for redistribution. The definitive version is published in ISS 2016, November 6-9, 2016, Niagara Falls, ON, Canada. $10.1145 / 2992154.2992158$
}

\section{ACM Classification Keywords}

H.5.2 Information interfaces and presentation (e.g., HCI): User Interfaces - Graphical user interfaces.

\section{INTRODUCTION}

Traffic congestion in major cities and highways is a growing problem in most countries. Perturbations such as accidents and breakdowns, or exceptional events such as demonstrations, can overload a road network that may already be operating at its limit, e.g. during rush hour. To prevent and to react efficiently to incidents and perturbations, road traffic in cities and highways is monitored in dedicated control centers.

Even for experienced operators, it is often challenging to evaluate the impact of an intervention on the network. While they are equipped with predefined traffic plans (sets of compatible interventions on a sector or area), it is still sometimes unclear which plan will work best for the current state of the network, in particular during exceptional events. This is where simulation models of road-traffic can help operators better understand and chose among possible intervention alternatives.

Road-traffic is a complex system with multiple agents (cars) that can behave in a non-deterministic manner. Researchers approximate road-traffic using methods from physics [16] or statistics and machine learning [43]. Their simulations can perform short-term traffic forecasting, identify problematic sectors with high-risk of traffic-congestion, and test new concepts to improve road-traffic such as dynamic adjustment of speed limits. Nevertheless, there is little work that looks at incorporating these results visually in traffic control centers.

Following interviews and observations of road-traffic control centers, we extracted user needs and designed a prototype system for road-traffic monitoring that runs on a touch en- 
abled ultra high-resolution wall display ${ }^{1}$. Taking advantage of their high resolution and large real-estate, our prototype extends the visualizations currently used in road-traffic control centers, to allow operators to concurrently explore and visualize results from multiple simulations testing alternative interventions on the network, both in a local and global scale.

We propose two techniques for viewing multiple simulations in combination with real traffic: (i) multiple views [21, 41, 45] to show the global state of the network; and (ii) DragMagic, a combination of DragMags [46] and magic lenses [8], to visualize localized sectors (Figure 1). We adapt and combine these techniques to visualize and compare several forecast visualizations using wall displays in control centers.

To better understand the performance of these techniques in our context, we compared them in a lab experiment. Participants performed well in comparison tasks of up to 6 different simulations, contrary to previous findings [30] that predicted decreased performance with the increase of comparisons. Our results also suggest that DragMagic is easier to master and may be beneficial when the number of simulations to compare is high, but that both techniques are viable alternatives. Early feedback on our prototype from experts also indicates a preference for DragMagic.

Our contributions are: (i) The user-centered design motivating a wall display prototype, building on interviews and observations of expert users, and the needs derived from them. (ii) Combining interaction and visualization techniques to present multiple simulated and real situations in the context of traffic management, something not seen in previous work. (iii) The first controlled study to evaluate these visualization techniques using an adapted situation-awareness task.

\section{BACKGROUND}

We discuss wall displays and Multi-Display Environments (MDE) for control rooms, and road traffic visualization.

\section{Wall Displays \& MDE for Control Rooms}

Control rooms are used to monitor complex systems like telescopes [28], power plants [26] or transportation networks [39]. They are usually equipped with a large display showing an overview of the situation and general information for all operators, and with individual workstations where operators act on the system (Figure 2).

Designers of such systems have recognized early on the importance of human factors and interaction in their work, and in particular the benefit of visualization walls and other shared displays. For instance, Smith et al. [38] study the control room of the London subway and highlight that a shared visualization can improve group awareness and communication in the team. And later, Starke et al. [39] studied how operators gathered and integrated information across displays in a simulated "object in the road" scenario.

Shared displays in control rooms are often treated as large monitors, and interaction is done using mice and keyboards.

\footnotetext{
1 an earlier version of the prototype alone has been presented in French as a Work in Progress (poster presentation) [31].
}

More recent work has focused on direct interaction with tabletops in crisis management $[32,13]$. Others have combined tabletops and tangible tools in power plant control centers [26], and tabletops and paper in air-traffic control [17]. Finally, recent work combined tabletops with shared vertical screens present in most control centers. For example, Bader et al. [5] combine a digital table map, a vertical screen with additional information, and a tablet to overlay personal information on the map. And Chokshi et al. [11] use tabletops to perform actions collaboratively on a central wall display.

In the above work, operators interact with the wall display indirectly, through mice/keyboards or tabletops. In few approaches, such as ours, interaction takes place directly on the wall displays. For example, in a scenario of police officers monitoring cars on a wall display, Ion et al. [19] use DragMags to focus on an area, with cut-offs of interesting items outside it connected with a folded strip. Our approach differs from previous work in control rooms. Beyond directly interacting with the wall display [19], we further propose using the wall display as a central interactive surface where operators can solve problems, not just monitor situations.

Irrespective of interaction platform, this previous work often relies on general purpose interaction and visualization techniques related to focusing and monitoring one or more areas of interest, such as space folding [14], magic lenses [8], DragMags [46] and multi-focus techniques [22, 25]. This is due to the need in such contexts to monitor several specific areas in detail, while still having an overview of the situation. For example Ion et al. [19] use DragMags with attached cut-offs; Schwarz et al. [37] use local semantic magnification with a Manhattan lens or a deported view on the wall, and multiple space-folds to visualize multiple areas with context-sensitive information at higher resolution $[9,37]$.

Our work poses visualization challenges that go beyond those seen in previous work, as it not only needs to allow operators to monitor multiple areas of interest simultaneously, but it also combines real data with simulation and forecasting visualizations. Thus the progress and results of one or even more simulations need to be viewed and understood concurrently with the actual road traffic data on multiple areas of interest.

\section{Road Traffic Visualization}

Trajectory visualization [2] focuses often on vessel traffic, marine $[23,34,36]$ and air [18, 35], and on an a-posteriori analysis of movement patterns over long time periods, using sophisticated interaction and visualization mechanisms such as aggregating paths, brushing and linking views, advanced statistics on selections, etc.

Similarly, most work on road traffic focuses on a-posteriori analysis and visualization of traffic patterns. For example, Andrienko et al. [1] extract and visualize meaningful places within movement data, and cluster spatio-temporal events or trajectories. Tominski et al. [40] visualize trajectories at different points in time, by stacking them on the 3rd dimension.

For real time traffic data visualizations, Wang et al. [44] visualize macro-traffic data recorded by transportation cells, using a combination of aggregated trajectories and of individ- 


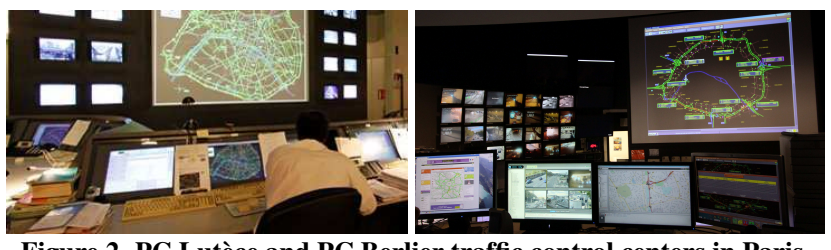

Figure 2. PC Lutèce and PC Berlier traffic control centers in Paris.

ual views for each cell showing vehicle animations. Nevertheless, the majority of modern traffic control centers, and studies conducted around them (see previous section), visualize real traffic data by coloring road segments based on traffic density or average vehicle speed, similarly to tools like Google Maps, Bing Maps, etc. As we saw, this visualization is often coupled with techniques that aid monitoring of different locations on wall displays, most notably variations of Magic lenses [8] and DragMags [30, 46].

We also use visualization lenses, but for a different purpose. As our goal is to augment real time traffic with results of simulations, lenses are used to provide side-by-side comparisons between the current situation and simulations in an area of interest. As an alternative to lenses, we also provide multiple juxtaposed views of the entire network [21, 41, 45], with real or simulated data. Another approach taken by Andrienko et al. [3] superimposed on a map the simulated results of road traffic bands in a time cube. Nevertheless, they focused on the simulation visualization and did not combine this visualization with real-time traffic. More recently, they presented a complete framework [4] to analyze road traffic, and model how additional cars will influence the network. To compare the impact of different possible interventions, they visualize results using difference maps, time cubes and statistics. Our work is orthogonal, as we focus on the user-centered design of a system to be incorporated in the shared wall of a control center, using visualizations to monitor multiple simulations and points of interest at a given time.

\section{OBSERVATIONS AND INTERVIEWS}

To understand the tasks, tools and needs of traffic operators, we visited two control centers: PC Lutèce that handles traffic for the city of Paris (Figure 2 left), and PC Berlier (Figure 2 right) that monitors the Périphérique, a motorway surrounding Paris. We observed two operators in PC Lutèce and interviewed one in depth (1h) as well as an operations engineer (1h); and we observed another two operators in PC Berlier and interviewed one $(1 / 2 \mathrm{~h})$ and the operator supervisor (1h).

General Observations. Both control centers are furnished with a large shared visualization wall showing the monitored network, surrounded by smaller screens with live camera feeds from the streets in PC Lutèce, and from the Périphérique and its tunnels in PC Berlier. Road segments are colored depending on traffic congestion from green (no congestion), to yellow, orange, and red (high congestion). Gray is used to indicate segments with faulty loop detectors. Arrows are used to highlight areas in which an intervention was done, either by the system (green arrow) or by an operator (yellow arrow if the system agrees with the intervention and red if it disagrees). Individual operator workstations are located in front of the wall, also displaying the network visualization (see Figure 2), alerts and other statistical information.

Due to the small scale and resolution of their monitors (w.r.t. the scale of the monitored network), operators tend to focus on localized areas of the network in their workstations, using mouse and keyboard to navigate. While they look at their individual workstations more, they all use the wall as an awareness monitor to acquire the "big picture" of the network state.

PC Lutèce. An automated system (SURF3) manages the traffic lights for approximately 1500 Parisian intersections, with more than 800.000 cars and 2.5 million pedestrian movements daily. It includes a library of "Traffic Light Plans" (a collection of consistent traffic light durations), and automatically choses the most appropriate plan, depending on the current traffic situation, the day of the week and the time of day. Under normal conditions (outside special events) the priority is public transport and pedestrian flow.

Operators can switch plans for specific sectors, or change traffic light duration of individual intersections for specific events or when incidents occur. Our interviewees explained that operators have a lot of experience in handling incidents in the city and can very accurately predict the impact of their actions and interventions in a local scale, such as a crossroad. Nevertheless, they explained it is difficult to access the impact of actions at a more global scale, e.g., it is often unclear how a change in a crossroad will impact other connected crossroads in the local sector or even the entire network.

PC Berlier. The center manages the traffic flow in the Périphérique motorway (IPER-REPER system), that hosts daily approximately 1.2 million car movements of commuters between Paris and its suburbs (60\% of traffic in the region, 2002). Operators have to constantly monitor traffic in the motorway and its tunnels in order to spot incidents and congestions. To optimize traffic flow, they can activate/deactivate lanes and reroute drivers using variable message signs, in particular since they face almost daily maintenance of lanes that requires reflow of traffic. To ensure safety in tunnels, operators can trigger and follow emergency plans, including evacuation, activation of smoke control systems, or closing tunnels in coordination with firefighter forces on the ground.

Our interviewees explained that a rerouting plan is in place for closing off sections of the motorway or tunnels. But they are hard pressed to apply it as it is difficult to assess the impact of such a drastic measure in each traffic situation. They described an incident with a tunnel flooded in both directions for 11 hours, where they considered applying this plan but could not risk it without a clear picture of potential global effects on the rest of the network.

The operator supervisor organizes shifts, oversees the good operation of the center and is involved in training new operators. She added that face the challenge of boredom: monitoring traffic feeds and messages from the public to detect incidents early is monotonous and operator attention can wander, and she felt that occasional task switching, such as forecast planning, could increase operator interest and focus. 
Other Control Centers. Road-traffic control centers around the world use different technologies and methods. In Paris, the traffic light control cycle is controlled by timing plans, while dynamic modification of the green light duration is not allowed. Other centers, like in London (SCOOT system ${ }^{2}$ ) allow such modifications. Interestingly, some centers started using predictive modeling to assess the impact of incidents and to help decision-making, such as in the Piemont Control Center in Italy (PTV Optima ${ }^{3}$ software). However, to our knowledge no control center combines forecasting and realtime traffic visualizations.

Motivation and User Needs. Our interviews and knowledge of existing centers suggest that: (i) It would be beneficial to incorporate visualization of predictive models with real-time monitoring tools, as the impact of actions is often hard to predict. (ii) Operators should be provided with likely outcomes of their interventions both globally on the entire network, and locally on specific sectors or intersections. (iii) Forecast visualizations increase the amount of information to be displayed, but are needed periodically, not on a constant basis. Given the advances in wall displays, i.e., their interactive support and their ability to display a large amount of information, wall displays can be a good platform for the next generation of road traffic systems, going beyond awareness monitors to also incorporate forecast analysis and visualization when needed.

Current control center setups also suffer from divided attention issues [37], with operators monitoring live camera feeds, the entire network on the visualization wall, and interacting through their individual workstations. If we consider additionaly visually displaying the results of predictive models, the already challenging task of monitoring real-time traffic can become increasingly difficult. As such, for our prototype we decided to show the visualization of the prediction models on the main visualization, and test the limits of how many such prediction models users can comfortably monitor.

\section{PROTOTYPE}

In order to explore solutions for integrating the results of forecasting models to real-time traffic monitoring, we implemented a set of visualizations and interaction techniques within a functional prototype. The prototype is developed using Java and the ZVTM Cluster library [29] that allows it to run simultaneously on desktops and on a wall driven by a computer cluster. Several desktop computers can share the view seen on the wall, but at different scales, as is currently done in control centers. On desktops, operators interact with mice and keyboard and their actions are mirrored on the wall.

On the wall we support two types of inputs: direct touch, and indirect touch using mobiles and tablets via the Smarties toolkit [10]. This dual input enables implicit zooming and context switching through movement [6,7], leverages the benefits of wall displays in high information density tasks [24], and provides new opportunities for collaborative data analysis [20]. Such interaction requires physical navigation

\footnotetext{
2 http://www.scoot-utc.com/ 3 http://vision-traffic.ptvgroup.com
}

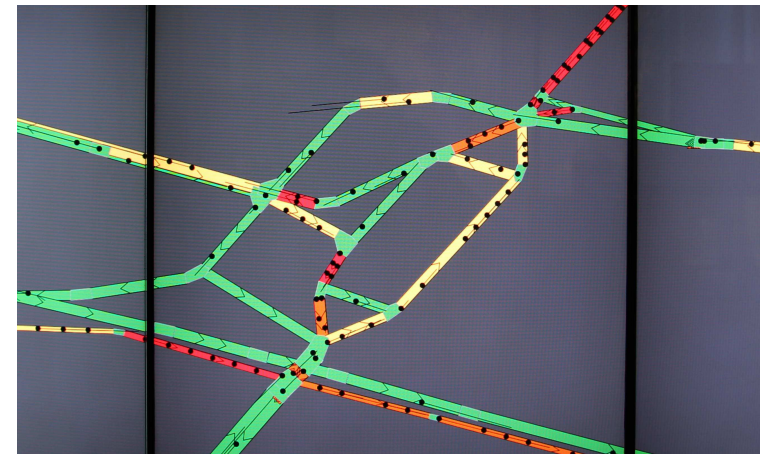

Figure 3. View of "Place de la Concorde" in Paris on our prototype.

[6], that could fatigue operators working long hours. We instead envision they'll be used occasionally: operators generally sit in front of their workstation, but when they address critical incidents or conduct planning sessions, they get up and interact with the wall. Focusing on a single shared screen could better support group work and awareness [15], and reduce the visual attention switch that occurs in MDE [33].

\section{Traffic data and modeling}

In our prototype, we represent each road network as a directed graph, with roads as links and intersections as nodes. The topography of existing road networks is extracted from OpenStreetMap data, or can be generated artificially (randomly) given a number of intersections and a desired road density.

Our system can process and display real-time streaming traffic-density data (e.g. data from the SURF3 system). To predict the evolution of current traffic, or of possible operator interventions, our prototype also models traffic. Roads are assigned speed limits, and intersection traffic-lights are assigned a duration. The duration of multiple lights (e.g. on a single road) can be synchronized as a group.

The current forecasting model is an extension of the Nagel and Schreckenberg one [27] developed by Chrobrok et al. [12]. It is based on cellular automata, and can model road networks with several lanes. At each intersection cars have a predefined probability of taking one of the available roads; this probability is calculated using real data, or the network topology favoring multi-lane roads.

A given state of the network can be cloned and used to run a forecasting model (accelerated) to (i) see a likely outcome of the current traffic, or (ii) see and compare the impact of possible interventions that adjust different parameters of the network (e.g. speed limit, lane closing, traffic light duration).

Our model is only a simplification of real road traffic, and more complex models have been developed in the field of traffic prediction using real-time data (e.g [4]). Nevertheless, our goal is not to develop a more accurate model, but rather to focus on the design of interaction and visualization techniques that can combine real time data and data from (multiple) forecasting simulations. Thus the traffic model is a plugin in our prototype, so as to be able to incorporate and test different models in the future. 

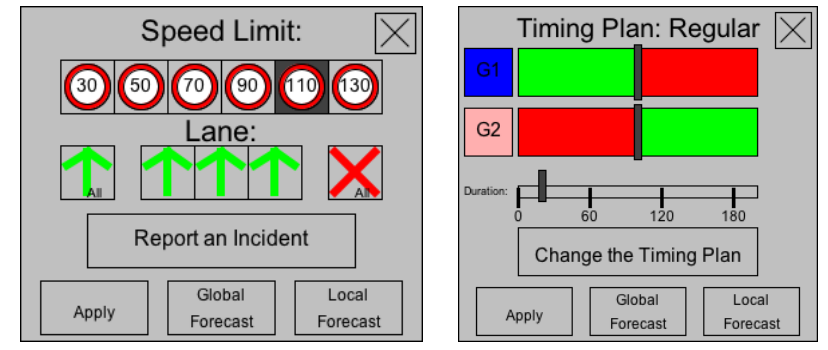

Figure 4. Context aware tool-palettes for modifying road (left) and intersection settings (right).

\section{Real Traffic, Visualization \& Interaction}

To visualize real-time data, we follow the conventions used in traffic control that operators are familiar with. Traffic density is represented by a progressive color scale: green (fluid), yellow, orange and red (saturated). Depending on data availability, individual cars can also be displayed as circles with a line representing their direction and speed vector (Figure 3).

Operators can invoke context-aware tool palettes (left click for mouse, long tap for touch) to manage roads or intersections. For a road they can alter the speed limit, open or close individual (or all) lanes, and report accidents (Figure 4-left). For an intersection, they can act on light duration: change the proportion of red/green light time, change the cycle duration, or change the current timing plan with another (Figure 4-right). These changes can either be applied immediately to the traffic, or clone the traffic and create a forecast visualization (discussed next).

Operator interventions on real traffic are reported on the map with arrows. By selecting an intervention arrow they can undo the action, or "lock" it so that it cannot be undone, suppressing the marker.

\section{Forecasts, Visualization \& Interaction}

We are interested in combining real traffic visualization, with likely forecasts of the potential future of this traffic, or of the impact of an intervention (e.g., closing a lane or changing light duration), calculated by traffic models. When operators invoke the tool palette to intervene on real traffic, instead of applying their changes, they can choose to instead start a forecast simulation. This clones the state of the real traffic and models the possible outcome of applying the changes, or the predicted outcome of the current situation sped-up.

Operators can intervene further on a forecast visualization, by changing road or intersection settings in the same way they do in the real-time visualization. They can choose to apply their changes to this particular forecast visualization, to the real traffic, or even clone the forecast simulation and apply the changes to the clone. Thus, they can generate multiple branching alternative simulations if desired.

As operators often want to compare the result of forecasts to the baseline traffic to see if there is an improvement, we use difference maps (as Lampe et al. [23]): colors do not indicate an absolute measure (e.g. density), but rather a positive or negative distance from a baseline situation (real traffic). The selection of an appropriate color map is important to highlight differences [42]. We use a diverging color scheme, adapted

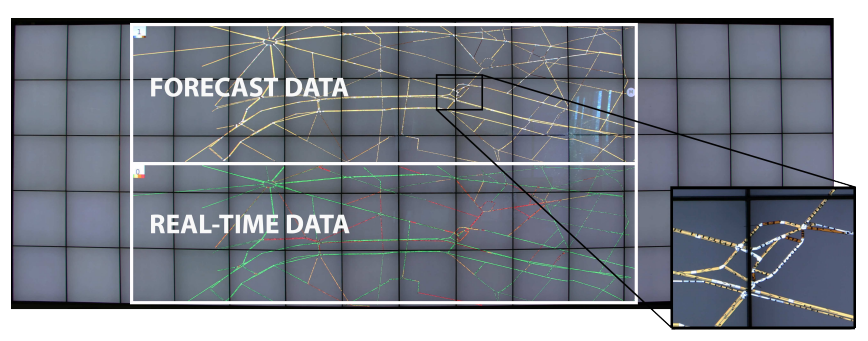

Figure 5. Global visualization of real traffic (bottom) and forecast (top) using MultiViews. A difference color map is used in forecast (cutout).

to be clearly visible on a wall (Figure 5 top \& Figure 6 DragMagic views). Three blue hues indicate improvement, three brown deterioration, and white color indicates a similar traffic density. These 7 colors represent all the possible amplitudes of the difference between real traffic and forecasts.

Based on our interviews, operators need to see two types of forecast results: Global, that show the forecast for the entire network; or Local, that are focused on a few neighboring roads and intersections, that we call an "area of interest".

Global (MultiViews). When operators are interested in forecast visualizations focusing on global outcomes, they can create a new view of the entire network for each simulation, following the idea of small multiples [21]. One view always represents the real time traffic, while the others are forecasts calculated by the predictive model (Figure 5).

Apart from using the tool palette, operators can also create new global forecast visualizations by tracing a vertical line inside a simulation to "split" its view, and create a perfect clone of it. Simulations are laid out on the wall using a grid optimization algorithm.

On the top left corner, global forecast visualizations have a unique identification number based on the order in which they were spawned, and a legend explaining the color range used in the view. They also have a button for invoking a tool palette, through which operators can change simulation settings, such as setting the prediction time frame with a slider from 0 to 30 minutes (a time duration considered to provide reliable results for our forecasting algorithm).

Local (DragMagic). When operators want to focus on particular areas of interest, they can invoke a variation of magic lenses $[8,25]$ that displays the forecast only for that area. This local view is placed at an offset position to avoid obscuring the real traffic at this area. A DragMagic can also be created by tracing a corner shaped gesture to define the area to be cloned in the DragMagic. As with a DragMag [46], the forecast visualization can be dragged, and is linked visually to the area of interest that is itself highlighted. Several forecast visualizations of different intervention simulations, focusing on the same area of interest, can be displayed side-by-side to show the possible outcomes (Figure 6).

Similarly to the global forecast visualization, DragMagics have a number identifying the simulation they are displaying, and a button to invoke the settings palette (Figure 6). This can be useful if operators want to monitor the results of a single simulation on more than one area of interest. It can be used 


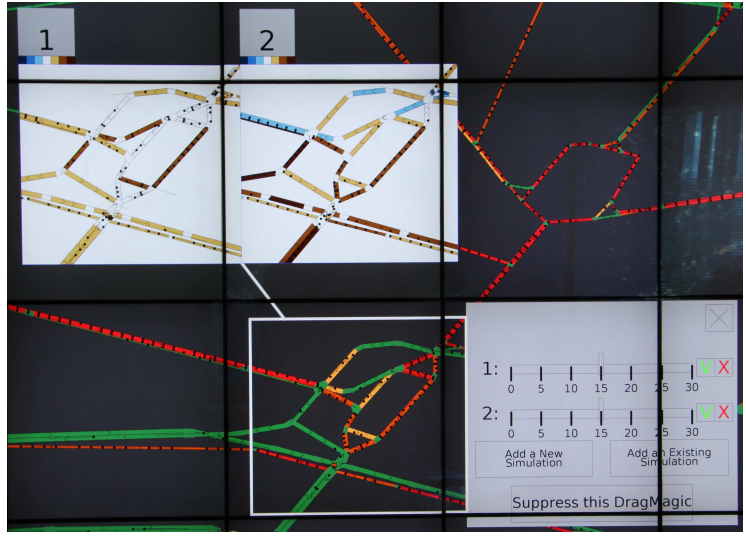

Figure 6. A DragMagic with two forecast visualizations (top left) linked to its area of focus (inside white rectangle), and its menu open (right).

for example to aid operators visualize the impact of an action on critical areas not directly linked to the location of the action, that may be far away. Such areas include vital pathways for access to hospitals, sensitive locations such as long tunnels, or central traffic hubs.

\section{MULTIPLE VIEWS VS. DRAGMAGIC}

An important and novel functionality of our prototype is the visualization of forecasts in combination with real traffic, using MultiViews to provide a global view of the models' prediction for the entire network, and DragMagic to visualize locally the predictions for specific areas.

MultiViews are well adapted for situations where operators need to see the impact for the entire network, as they show global forecasts. When they are interested in a single small area of the network, DragMagics are better for showing local effects. However, the situation is more complex when operators need to consider several areas of interest (critical areas) on the network. Due to the higher number and sparsity of areas of interest, this task is neither clearly local nor global, and thus it is unclear which technique fares best. DragMagic likely works well for few areas of interest, but as their number increases they approximate the entire network, and as such MultiViews may be better. Moreover, it is unclear how hard it is to follow multiple simulations running at the same time in order to decide between alternatives, using either technique. We thus designed an experiment to compare viewers' performance using DragMagic and MultiViews for this intermediate case, varying the number of simulations and areas of interest.

Our factors are: two techniques TECH, DragMagic and MultiViews; number of simulations \#SIMU, with 3 values $\{2,4,6\}$ (to simplify, we consider real-time traffic as a simulation); and number of areas of interest \#AOI, with 3 values $\{3,5,7\}$.

In a trial, we showed participants several traffic simulations, where one ("simulation 0") is considered the reality and is coded with the classic red to green color coding. The rest use difference maps with simulation 0 (see Prototype). In MultiViews, on each simulation the areas of interest are highlighted using white rectangles. In DragMagic only simulation 0 is shown fully, and a DragMagic per area of interest is used to display the remaining simulations. For consistency, in the ex- periment all areas of interest contained exactly two roads, and simulations were updated every 10 seconds.

The layout of MultiViews was such that simulations had the same size, and were as big as possible while fitting on the wall. DragMagics were positioned such that they were as close as possible of their area of interest, while not overlapping with other areas of interest or DragMagic.

In each trial, participants were asked four questions, separated by intervals of about $30 \mathrm{sec}$. Two questions were on the present state of the areas of interest, and two on their past history (inspired by tests accessing situation awareness that alternate and repeat questions on present and past, as in [19]):

$\mathrm{Q}_{\text {pres }}$ "At the present moment, which simulation is the best for the areas of interest?". Asked 1st and 3rd.

$\mathrm{Q}_{\text {hist }}$ "From the beginning of this trial, which simulation was the best for the areas of interest?". Asked 2nd and 4th.

When it was time for a question, an alarm rung, the simulations paused, and the question was displayed at the top of the wall. Participants gave their answer (the simulation number) using a smartphone. They were instructed to be as fast as possible while minimizing errors. We explicitly told participants not to perform a detailed comparison, but to give us their overall impression, especially for $\mathrm{Q}_{\text {pres }}$ where a detailed comparison is tedious but possible. Trials lasted 2 minutes plus the time taken by participants to answer the questions.

Our first working hypothesis is that:

H1 DragMagic will perform better than MultiViews, as viewers have to monitor a smaller area.

Following Plumlee and Ware [30] that link performance with the number of visual comparisons in a task, it is reasonable to hypothesize that increasing the number of simulations \#SIMU and areas of interest \#AOI will decrease performance overall. However, our experiment was not designed to evaluate the effects of \#SIMU and \#AOI, but rather to evaluate a possible interaction of TECH with \#SIMU and \#AOI. According to Plumlee and Ware [30] distance between comparisons can also deteriorate performance:

H2 DragMagic will perform better with a larger number of simulations \#SIMU as the distance of the areas being compared is reduced; while MultiViews will perform better with more areas of interest \#AOI, as its global view will be a good approximation of all the \#AoI's.

\section{Experimental Design}

Participants. Sixteen volunteers took part in the experiment ( 8 female, 8 male), aged 23 to 32 , with normal or correctedto-normal vision. As participants needed to tell the difference between several shades of the same color, they took the Ishihara Color Blindness test before the experiment to ensure they did not suffer from color-blindness. As our experiment is perceptual in nature (tracking of color changes over time) with no domain knowledge requirements, we believe that designing with experts and experimenting initially with non-experts is valid for measuring perceptual situation awareness (similarly to previous work, e.g., [19]). 


\begin{tabular}{|l|rrrr|}
\hline Effect for Time & $n, d$ & $\mathrm{~F}_{n, d}$ & \multicolumn{1}{c|}{$p$} & \\
\hline TECHORDER & 1,14 & 2.48 & 0.137 & \\
TECH & 1,14 & 5.28 & 0.038 & $\star$ \\
\#SIMU & 2,28 & 31.8 & $<0.001$ & $\star$ \\
\#AOI & 2,28 & 3.77 & 0.036 & $\star$ \\
TECHORDER $\times$ TECH & 1,14 & 6.44 & 0.024 & $\star$ \\
TECHORDER & 2,28 IMU & 4.20 & 0.025 & $\star$ \\
TECHORDERX\#AOI & 2,28 & 0.56 & 0.565 & \\
TECHX\#SIMU & 2,28 & 2.61 & 0.091 &. \\
TECHX\#AOI & 2,28 & 0.44 & 0.650 & \\
\#SIMUX\#AOI & 4,56 & 5.38 & $<0.001$ & $\star$ \\
\hline
\end{tabular}

(a)
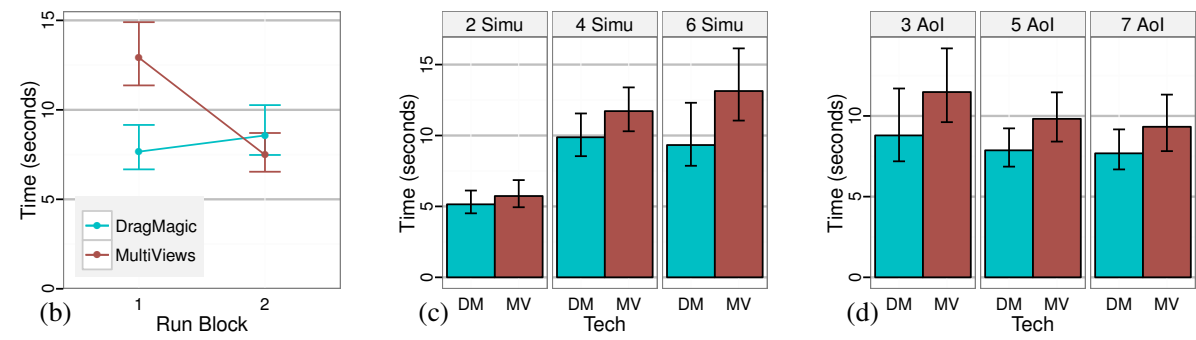

Figure 7. Time to answer for question $\mathbf{Q}_{\text {pres }}$ : (a) Results of the degree 2 ANOVA in the mixed model TECHORDER $\times$ TECH $\times \#$ SIMU $\times \#$ AOI $\times$ Rand(PARTICIPANT) (there is no significant interaction of degree $>2$, all $p$ ' $s>0.4)$; statistically significant results $(p<.05)$ are starred, whereas a dot marks statistical trends $(p<.1)$. (b) Time by TECH depending on whether a technique was run in the $1^{\text {st }}$ block or in the $2^{\text {nd }}$ one. (c) Time for each TECH by \#SIMU. (d) Time for each TECH by \#AOI. In (c) and (d), DM stands for DragMagic and MV for MultiViews.

Apparatus. We used an interactive wall $(5.9 \mathrm{~m} \times 1.96 \mathrm{~m}$ wide, with a resolution of $14400 \times 4800$ pixels), made of 75 LCD displays (21.6 inches, $3 \mathrm{~mm}$ bezels each), seen in Figure 1. The wall was driven by a rendering cluster of 10 computers. The experimental software, built on our prototype, ran on a master machine connected to the cluster through $1 \mathrm{Gbit}$ Ethernet. Participants answered questions using a smartphone.

Procedure and Design. The experiment is a $[2 \times 3 \times 3]$ withinparticipants design with factors TECH (MultiViews and DragMagic), \#SImu (2, 4 and 6) and \#AOI (3, 5 and 7). We blocked by TECH and the order was counter-balanced between participants: half started with DragMagic and half with MultiViews. For each TECH, a first trial was used to explain the task, questions and the visualization (e.g., color code, areas of interest, simulation). Then participants conducted 3 training trials before proceeding to the $9(=3 \times 3)$ measured trials. For these 9 trials, the \#SIMU increased gradually (first 2, then 4 and 6). And for each simulation number condition, the \#AOI also increases gradually (3, then 5 and 7). We started with the a-priori easier tasks to try to reduce learning effects.

The network used are the main roads in Paris city center. Using our prototype we built 13 sets of simulations (4 for the training and 9 for the measured trials), by generating a large number of 2 min simulations and selecting ones with a similar number of color changes (about 1000 per simulation). Real traffic ( $\operatorname{sim} 0)$ was also generated this way for consistency. To ensure a fair comparison across techniques, we took the original simulation sets, and built another 13 ones by changing the simulations order. TECH presentation order was counterbalanced consistently with the sets.

Sessions lasted 1 hour, and at the end participants completed a questionnaire on strategy, workload (customized NASA TLX questionnaire) and preference.

Measures. We recorded the time to answer the questions Time, and participants' answers. Time is important in a control room context, as operators need to evaluate situations and act quickly. In our experiment, slower answer times can indicate that in some conditions assessing a situation is harder and requires more reflection. When two or more simulations are displayed, a given imperfect answer could be better than another, as simulations have an order when it comes to improvement over the real situation. Thus, we define NError as: $(R-1) /(\#$ S imu -1$)$ where $R$ is the rank of the simula- tion when ordered from best to worst. Using \#SIMU ensures we normalize the error per number of simulations. We also report on absolute number of errors per condition (Err).

\section{Results}

We first look at the results for $\mathrm{Q}_{\text {pres }}$, the results for $\mathrm{Q}_{\text {hist }}$ are similar and discussed next. We noticed that the presentation order of TECH has an impact on the results, thus we report on the between-subject factor TECHORDER. Error bars in our images represent $95 \%$ CI computed with all the data points using $\mathrm{BCa}$ bootstrapping. For post-hoc tests we use paired t-test with Holm correction.

\section{Time}

Figure 7-a shows the detailed ANOVA for Time. TECH has a significant effect on Time (DragMagic being faster), but we also have a significant TECH $\times$ TECHORDER interaction. A post-hoc test shows that DragMagic is significantly faster than MultiViews ( $p=0.007$, a speed-up of 33\%) for participants starting with MultiViews, but not for participants starting with DragMagic ( $p=0.753$, almost the same mean Time). See Figure 7-b. Thus, $H 1$ is only satisfied when participants are mastering the techniques and task.

Note that the Time for DragMagic for both TECHORDERs, and for MultiViews when seen second are very similar, and all three significantly faster than MultiViews when seen first ( $p$ 's $<0.009)$. We see a positive learning transfer from DragMagic to MultiViews, while the time for DragMagic is similar irrespective of order indicating it is easier to master.

There is a significant effect of \#SIMU and \#AOI on Time, with significant interactions TECHORDER $\times \#$ SIMU and \#SIMU $\times$ \#AOI, but no significant interaction with TECH (next paragraph). Post-hoc tests show that participants were overall faster with 2 simulations than with 4 and 6 ( $p$ 's $<0.001$, no significant difference between 4 and 6 simulations, $p=0.648$; see Figure 7-c). When starting with MultiViews this difference between 2 simulations and 4 or 6 simulations are bigger than when starting with DragMagic (the TECHORDER $\times \#$ SIMU interaction). Surprisingly, when it comes to \#AOI, participants were overall significantly slower with 3 AoIs than with 7 AoIs ( $p=0.025$; no other significant difference between the AoIs; see Figure 7-d). This difference is mainly caused by the case of 6 simulations (the \#SIMU $\times \#$ AOI interaction) and suggests that participants were able to use the time in between questions to select and focus on a few promising simulations, reducing the number of comparisons at answer time. 
The TECH $\times$ \#SIMU interaction is not significant $(p=0.091)$. However, the difference between the better performance of DragMagic over MultiViews grows with \#SIMU and becomes significant with 6 simulations: $5.1 s$ vs. $5.7 s$ for 2 simulations $(p=0.466), 9.9 s$ vs. $11.7 s$ for $4(p=0.146)$, and $9.3 s$ vs. $13.1 s$ for 6 simulations $(p=0.038)$. Thus, the first part of $H 2$ is partially confirmed. Results do not confirm the second part of $H 2$, as there is no effect of \#AOI on TECH.

\section{Normilized Errors}

Regarding normalized errors, the only significant result is an effect of \#SIMU $\left(F_{2,28}=7.48, p=0.002\right)$. Participants made significantly more errors with 4 simulations (on average 0.13 ) than with 2 simulations (on average $0.06, p=0.02$. And trend for more errors with 4 simulations than with 6 simulations (average of $0.08, p=0.065$ ). We note that statistical trends with absolute number of errors are similar, with the additional difference between 2 and 6 simulations $(p<.001)$. Mean absolute error was $0.06,0.27,0.20$ for 2,4 , and 6 simulations respectively.

An important remark is that DragMagic and MultiViews exhibit very similar average normalized error, overall (0.087 vs. 0.086), and also depending on whether they are seen first or second (0.106 vs. 0.097 for block one and 0.068 vs. 0.076 for block two). The same holds for absolute error (0.18 vs. 0.185 ). Thus, the above results on Time cannot be attributed to a speed-accuracy trade-off.

The $Q_{\text {hist }}$ Question vs. the $Q_{\text {pres }}$ Question

Result trends for $\mathrm{Q}_{\text {hist }}$ are very similar to $\mathrm{Q}_{\text {pres }}$, we thus omit a detailed presentation of the results. For instance, we have a significant TECHORDER $\times$ TECH interaction $\left(F_{1,14}=5.43\right.$, $p=0.035)$, DragMagic is significantly faster than MultiViews for the participants starting with MultiViews ( $p=0.002$, a $26 \%$ speed-up), but not for the participants starting with DragMagic. Moreover, the Time for DragMagic for both TECHORDER and MultiViews for the participants starting with DragMagic are very close. Average errors are almost the same for the 4 conditions considered above.

Participants were overall significantly faster with $\mathrm{Q}_{\text {pres }}$ than with $\mathrm{Q}_{\text {hist }}(p<0.001,6.5 s$ vs. 9.2s), and made significantly less errors ( $p<0.001,0.09$ vs. 0.25$)$. This is a reasonable result as $\mathrm{Q}_{\text {hist }}$ is more complex since it relies more heavily on memory.

\section{Subjective Results}

Eleven out of sixteen participants preferred to use DragMagic over MultiViews, a slight - non significant - preference for DragMagic $\left(\chi_{1,16}^{2}=2.25, p=0.134\right)$. Seven out of the eight participants that started with MultiViews preferred DragMagic $\left(\chi_{1,8}^{2}=4.5, p=0.034\right)$, while from the participants that started with DragMagic, four indicate a preference for DragMagic and four for MultiViews. Thus, TECH preference matches closely the results on time.

Regarding subjective mental workload, a TECHORDER $\times$ TECH interaction is again present. Participants starting with MultiViews reported a significantly higher mental workload for MultiViews than for DragMagic ( $p=0.008,4.8$ vs. 3.9 on a 1-7 scale). While for participants starting with DragMagic reported mental workload was similar between MultiViews (4.2) and DragMagic (4.1).
When reporting strategies, 10 participants explicitly mentioned they always chose 2-3 promising simulations to focus on, even when more simulations were running.

\section{Summary and Discussion}

Participants starting with MultiViews were slower with this technique (without making less errors): participants starting with DragMagic were $33 \%$ faster with both DragMagic and with MultiViews, when compared to participants that started with MultiViews. The speed of DragMagic was fairly consistent across ordering conditions. The subjective results (preference and mental workload) show a similar trend. Even if there is a learning effect on MultiViews, there is no such effect on DragMagic, which suggests that participants mastered the use of DragMagic faster than MultiViews. Moreover, using DragMagic has a positive learning impact on MultiViews.

DragMagic exhibits slightly better performance than MultiViews as the number of simulations increases, indicating that, as expected, reducing the distance between the simulations to be compared can be beneficial. Nevertheless, we did not measure any difference between MultiViews and DragMagic when the number of areas of interest increased.

Contrary to the model of Plumlee and Ware [30], we do not have a clear growing relation between Time and the number of comparisons needed to perform the task, in particular when it comes to the increase of areas of interests and number of simulations. This can be explained by the temporal nature of our task. Based on their comments, participants continuously compared simulations in the time between questions, not just at question time, and were thus able to identify and ignore ahead of time non-promising simulations, providing answers more quickly. Thus, the Plumlee and Ware model does not extend to tasks that have a temporal continuity.

When considering the traffic control context, our results indicate that both techniques can be effective for comparison of simulation results of possible interventions, without a strong performance difference once users become familiar with them. We feel this shows both designs as viable alternatives in terms of performance, and thus designers can choose based on other criteria, like space requirements, positioning of operators in the control room, areas to be monitored, etc.

Our study is perceptual in nature, thus we felt 16 non-expert participants were appropriate. Nevertheless, a larger number of participants could have provided more power to our results. Moreover, our study did not evaluate the interactive aspect of creating, managing and rearranging the DragMagic or the MultiViews. Finally, to ensure a realistic experiment duration, we fixed the time interval and changes between questions, but verifying that our results hold under varying intervals would strengthen our findings. These remain future work.

\section{EARLY FEEDBACK FROM EXPERTS}

A user feedback session was conducted with three of our original users, using a combination of a desktop demo and a video of the prototype used on the wall.

Our interviewees found the idea of interleaving the results of real time traffic data and model predictions very useful. How- 
ever, all explained that these visualizations would not be used constantly, rather occasionally in situations when the results of possible actions are hard to predict. Operators mentioned they would most likely interact with them from their workstations. Nevertheless, the operations manager explained that the setup of the walk-up and use wall (away from their workstation) could benefit operators in two ways: first by helping them focus on the task at hand without distractions such as camera feeds, etc. And second it could shift their attention away from the monotonous monitoring tasks, and thus alleviating boredom and improving overall performance.

All interviewees seemed to be more interested in the DragMagic visualization for comparing real and forecast data, as they give operators the information they need in the areas of interest "and also the state of the traffic around it".

Two operators thought separately of another use for our system not envisioned before. They felt our techniques can help them diagnose and predict problematic situations by comparing "benchmark" traffic data (past data recorded under normal conditions) with current traffic. As one explained, the system could suggest to operators to open the comparative visualizations when a big enough difference is detected. The visualizations could then provide context and help operators determine if the unusual behavior is a potential unreported incident that requires further investigation, or if the traffic situation is deteriorating and requires intervention.

They also highlighted the need to incorporate some additional functionality, such as the ability to update messages on electronic signs around the city and motorway for the public, and to integrate multiple global traffic light timing plans used currently in the city that we did not have access to initially.

One operator and the operations engineer are currently involved in the development of new systems that may include predictive models (without visualization). Both explained that our setup could also be very useful to their colleagues that work on improving traffic modeling for control centers. Their algorithms require careful tuning and they often need to run multiple small variations of them, that are hard to visualize concurrently on desktop screens.

\section{CONCLUSION}

We propose using interactive wall displays in road-traffic control centers for interacting with real-time and simulated traffic data. After visiting two such centers, we designed a prototype that allows to monitor and act on the traffic (or on simulations) and, more importantly, to compare real traffic and several forecast simulations. To this end we use two visualization techniques: MultiViews and DragMagic, that we compared in a lab experiment in terms of situation awareness.

The results show that DragMagic is easier to master, but that both techniques are reasonable design options for control centers, even for several simulations and areas of interest. It seems that the speed of monitoring tasks, that are temporal in nature, is not drastically affected by the number of comparisons in multiple views (predicted by the Plumlee and Ware model [30]). Viewers can identify and ignore non-promising forecasts, reducing the number of effective comparisons. Revising such models is interesting future work.

Expert users provided encouraging feedback and suggestions after seeing the prototype, appreciating in particular the use of DragMagic to follow forecast simulations while keeping the context of real traffic. They also found the prototype useful to compare real and past data to help identify possible problematic situations.

We next plan to study collaboration in control centers, in particular the use of several DragMagics, and cases where some operators interact with the wall and others with their workstation, a functionality we already support.

\section{ACKNOWLEDGMENT}

We thank P. Goatin, L. Charansonney and N. Acherar for their availability. We also thank C. Appert, E. Pietriga, O. Gladin and the members of the ILDA team. This research was partially supported by EquipEx Digiscope (ANR-10-EQPX-26-01).

\section{REFERENCES}

1. Andrienko G., Andrienko N., Hurter C., Rinzivillo S. \& Wrobel S. From movement tracks through events to places: Extracting and characterizing significant places from mobility data. VAST'11, IEEE (2011), 161-170.

2. Andrienko N. \& Andrienko G. Visual analytics of movement: An overview of methods, tools and procedures. Info. Vis. 12, 1 (2013), 3-24.

3. Andrienko N., Andrienko G. \& Rinzivillo S. Exploiting spatial abstraction in predictive analytics of vehicle traffic. ISPRS Int. J. Geo-Inf. 4, 2 (2015), 591-606.

4. Andrienko N., Andrienko G. \& Rinzivillo S. Leveraging spatial abstraction in traffic analysis and forecasting with visual analytics. Info. Sys. 57 (2016), 172-194.

5. Bader T., Meissner A. \& Tscherney R. Digital map table with fovea-tablett $\AA$ : Smart furniture for emergency operation centers. ISCRAM '08, ISCRAM (2008), 2033-2034.

6. Ball R., North C. \& Bowman D. A. Move to improve: promoting physical navigation to increase user performance with large displays. CHI '07, ACM (2007), 191-200.

7. Bezerianos A. \& Isenberg P. Perception of visual variables on tiled wall-sized displays for information visualization applications. IEEE TVCG 18, 12 (2012), 2516-2525.

8. Bier E. A., Stone M. C., Pier K., Buxton W. \& DeRose T. D. Toolglass and magic lenses: The see-through interface. SIGGRAPH '93, ACM (1993), 73-80.

9. Butscher S., Hornbæk K. \& Reiterer H. Spacefold and physiclenses: Simultaneous multifocus navigation on touch surfaces. AVI '14, ACM (2014), 209-216.

10. Chapuis O., Bezerianos A. \& Frantzeskakis S. Smarties: An input system for wall display development. $\mathrm{CHI}$ '14, ACM (2014), 2763-2772.

11. Chokshi A., Seyed T., Marinho Rodrigues F. \& Maurer F. ePlan multi-surface: A multi-surface environment for 
emergency response planning exercises. ITS '14, ACM (2014), 219-228.

12. Chrobok R., Hafstein S. F. \& Pottmeier A. Olsim: A new generation of traffic information systems. Forschung und wissenschaftliches Rechnen, GWDG-Berichte Nr. 63 (2004), 11-25.

13. Doeweling S., Tahiri T., Sowinski P., Schmidt B. \& Khalilbeigi M. Support for collaborative situation analysis and planning in crisis management teams using interactive tabletops. ITS '13, ACM (2013), 273-282.

14. Elmqvist N., Henry N., Riche Y. \& Fekete J.-D. Melange: Space folding for multi-focus interaction. $\mathrm{CHI}$ '08, ACM (2008), 1333-1342.

15. Gutwin C. \& Greenberg S. A descriptive framework of workspace awareness for real-time groupware. JCSCW 11, 3 (2002), 411-446.

16. Helbing D. Traffic and related self-driven many-particle systems. Rev. Mod. Phys. 73, 4 (2001), 1067-1141.

17. Hurter C., Lesbordes R., Letondal C., Vinot J.-L. \& Conversy S. Strip'TIC: Exploring augmented paper strips for air traffic controllers. AVI'12, ACM (2012), $225-232$.

18. Hurter C., Tissoires B. \& Conversy S. Fromdady: Spreading aircraft trajectories across views to support iterative queries. IEEE TVCG 15, 6 (2009), 1017-1024.

19. Ion A., Chang Y.-L. B., Haller M., Hancock M. \& Scott S. D. Canyon: Providing location awareness of multiple moving objects in a detail view on large displays. $\mathrm{CHI}$ '13, ACM (2013), 3149-3158.

20. Jakobsen M. R. \& Hornbæk K. Up close and personal: Collaborative work on a high-resolution multitouch wall display. ACM ToCHI 21, 2 (2014), 11:1-11:34.

21. Javed W. \& Elmqvist N. Exploring the design space of composite visualization. PacificVis '12, IEEE (2012), $1-8$.

22. Javed W., Ghani S. \& Elmqvist N. Polyzoom: Multiscale and multifocus exploration in 2D visual spaces. CHI '12, ACM (2012), 287-296.

23. Lampe O. D., Kehrer J. \& Hauser H. Visual analysis of multivariate movement data using interactive difference views. $V M V$ '10, EG (2010), 315-322.

24. Liu C., Chapuis O., Beaudouin-Lafon M., Lecolinet E. \& Mackay W. Effects of display size and navigation type on a classification task. CHI' 14 , ACM (2014), 4147-4156.

25. Lobo M.-J., Pietriga E. \& Appert C. An evaluation of interactive map comparison techniques. $C H I$ ' 15 , ACM (2015), 3573-3582.

26. Müller J., Schwarz T., Butscher S. \& Reiterer H. Back to tangibility: A post-wimp perspective on control room design. AVI '14, ACM (2014), 57-64.

27. Nagel K. \& Schreckenberg M. A cellular automaton model for freeway traffic. J. Phys. I 2, 12 (1992), 2221-2229.

28. Pietriga E., et al. Interaction design challenges and solutions for ALMA operations monitoring and control.
In SPIE 8451, Software and Cyberinfrastructure for Astronomy II, SPIE (2012), 10:1-10:16.

29. Pietriga E., Huot S., Nancel M. \& Primet R. Rapid development of user interfaces on cluster-driven wall displays with jBricks. EICS '11, ACM (2011), 185-190.

30. Plumlee M. D. \& Ware C. Zooming versus multiple window interfaces: Cognitive costs of visual comparisons. ACM ToCHI 13, 2 (2006), 179-209.

31. Prouzeau A., Bezerianos A. \& Chapuis O. Surveillance du trafic routier avec un mur d'écrans. IHM TeC '15, ACM (2015), 6 pages.

32. Qin Y., Liu J., Wu C. \& Shi Y. uEmergency: A collaborative system for emergency management on very large tabletop. ITS '12, ACM (2012), 399-402.

33. Rashid U., Nacenta M. A. \& Quigley A. Factors influencing visual attention switch in multi-display user interfaces: A survey. PerDis '12, ACM (2012), 1:1-1:6.

34. Riveiro M. Evaluation of normal model visualization for anomaly detection in maritime traffic. ACM TIIS 4, 1 (2014), 5:1-5:24.

35. Scheepens R., Hurter C., van de Wetering H. \& van Wijk J. Visualization, selection, and analysis of traffic flows. IEEE TVCG 22, 1 (2016), 379-388.

36. Scheepens R., Willems N., van de Wetering H., Andrienko G., Andrienko N. \& van Wijk J. J. Composite density maps for multivariate trajectories. IEEE TVCG 17, 12 (2011), 2518-2527.

37. Schwarz T., Butscher S., Mueller J. \& Reiterer H. Content-aware navigation for large displays in context of traffic control rooms. AVI'12, ACM (2012), 249-252.

38. Smith P., Blandford A. \& Back J. Questioning, exploring, narrating and playing in the control room to maintain system safety. Cognition, Technology \& Work 11, 4 (2009), 279-291.

39. Starke S., Cooke N., Howes A., Morar N. \& Baber C. Visual sampling in a road traffic management control room task. In International Conference on Ergonomics \& Human Factors, Taylor \& Francis (2015), 503-511.

40. Tominski C., Schumann H., Andrienko G. \& Andrienko N. Stacking-based visualization of trajectory attribute data. IEEE TVCG 18, 12 (2012), 2565-2574.

41. Tufte E. R. The Visual Display of Quantitative Information. Graphics Press, Cheshire, CT, USA, 1986.

42. Verma V. \& Pang A. Comparative flow visualization. IEEE TVCG 10, 6 (2004), 609-624.

43. Vlahogianni E. I., Golias J. C. \& Karlaftis M. G. Short-term traffic forecasting: Overview of objectives and methods. Transport reviews 24, 5 (2004), 533-557.

44. Wang Z., Ye T., Lu M., Yuan X., Qu H., Yuan J. \& Wu Q. Visual exploration of sparse traffic trajectory data. IEEE TVCG 20, 12 (2014), 1813-1822.

45. Wang Baldonado M. Q., Woodruff A. \& Kuchinsky A. Guidelines for using multiple views in information visualization. AVI '00, ACM (2000), 110-119.

46. Ware C. \& Lewis M. The dragmag image magnifier. CHI'95, ACM (1995), 407-408. 